COMMUNICATIONS IN

ANALYSIS AND GEOMETRY

Volume 15, Number 1, 121-146, 2007

\title{
Non-negatively curved Kähler manifolds with average quadratic curvature decay
}

\author{
Albert Chau $^{1}$ And Luen-Fai TAm ${ }^{2}$
}

Let $(M, g)$ be a complete noncompact Kähler manifold with non-negative and bounded holomorphic bisectional curvature. Extending our techniques developed in [A. Chau and L.-F. Tam. On the complex structure of Kähler manifolds with non-negative curvature, J. Differs. Geom. 73 (2006), 491-530.], we prove that the universal cover $\widetilde{M}$ of $M$ is biholomorphic to $\mathbb{C}^{n}$ provided either that $(M, g)$ has average quadratic curvature decay, or $M$ supports an eternal solution to the Kähler-Ricci flow with non-negative and uniformly bounded holomorphic bisectional curvature. We also classify certain local limits arising from the Kähler-Ricci flow in the absence of uniform estimates on the injectivity radius.

\section{Introduction}

Generalizing the classical uniformization theorems to higher dimensions is a central problem in the study of complex manifolds. It is a particularly interesting problem on complete Kähler manifolds. In this paper, we are interested in complete noncompact Kähler manifolds with positive curvature. For such manifolds, there is a well-known conjecture by Yau [36] which states that a complete noncompact Kähler manifold with positive holomorphic bisectional curvature is biholomorphic to $\mathbb{C}^{n}$. Yau's conjecture in its full generality remains unsolved. The first major results supporting the conjecture were obtained by Mok et al. [22], and the conjecture has since been studied extensively, see $[7,8,9,11,12,21,23,26,28,33,34]$. Recently in $[9]$ the authors proved the following.

Theorem 1.1. Let $\left(M^{n}, \widetilde{g}\right)$ be a complete noncompact Kähler manifold with non-negative and bounded holomorphic bisectional curvature and maximal volume growth. Then $M$ is biholomorphic to $\mathbb{C}^{n}$.

\footnotetext{
${ }^{1}$ Research partially supported by NSERC grant no. \# 327637-06.

${ }^{2}$ Research partially supported by Earmarked Grant of Hong Kong \#CUHK403005.
} 
Here, maximum volume growth means that

$$
\operatorname{Vol}(B(p, r)) \geq C_{1} r^{2 n} ; \quad \forall r \in[0, \infty)
$$

for some $C_{1}>0$ and $p \in M$. This assumption of maximum volume growth is rather strong. Consider the following average quadratic curvature decay condition

$$
\frac{1}{V_{x}(r)} \int_{B_{x}(r)} R \leq \frac{C_{2}}{1+r^{2}}
$$

for some $C_{2}>0$, all $x \in M$ and all $r>0$. Here, $B_{x}(r)$ is the geodesic ball around $x$ with radius $r$ and volume $V_{x}(r)$ and $R$ is the scalar curvature of $M$. It was conjectured by Yau that if (1.1) is true, then (1.2) will be satisfied automatically for a complete noncompact Kähler manifold with nonnegative holomorphic bisectional curvature. Provided that the curvature is bounded, this was recently confirmed by $\mathrm{Ni}$ [25] (this was earlier confirmed by Chen-Tang-Zhu [11] for the case of dimension 2 and Chen-Zhu [13] in all dimensions under the additional condition that the curvature operator is non-negative). In general, (1.2) does not imply (1.1). For example, let $M$ satisfy (1.2) and let $M_{1}$ be the product of $M$ and a flat torus. Then $M_{1}$ also satisfies (1.2). But $M_{1}$ does not have maximal volume growth. However, it is an open question whether (1.2) will imply (1.1) under the additional assumption that $M$ has positive bisectional curvature.

In $[10,22,23,26,28]$ without assuming the maximum volume growth condition, it was proved that if $R$ decays faster than quadratic, then the manifold $M$ with non-negative holomorphic bisectional curvature must be flat. Hence one would expect that Theorem 1.1 is still true if the maximum volume growth condition is removed and is replaced by the weaker condition (1.2). In this work, we confirm this expectation in the following:

Theorem 1.2. Suppose $\left(M^{n}, g\right)$ has holomorphic bisectional curvature which is bounded, non-negative and has average quadratic curvature decay. Then $M$ is holomorphically covered by $\mathbb{C}^{n}$.

As in [9], we will use the Kähler-Ricci flow:

$$
\left\{\begin{array}{l}
\frac{\partial}{\partial t} \tilde{g}_{i \bar{\jmath}}(x, t)=-\tilde{R}_{i \bar{\jmath}}(x, t) ; \\
\tilde{g}_{i \bar{\jmath}}(x, 0)=g_{i \bar{\jmath}}(x) .
\end{array}\right.
$$

The main difficulty in proving Theorem 1.2 with the methods in [9] is the lack of good lower bound for the injectivity radius of $\tilde{g}(t)$. Indeed, 
Theorem 1.2 was proved by the authors in [9] under the additional assumption that the curvature operator is non-negative, in which case a good lower bound on the injectivity radius can be obtained. Such lower bounds can also be obtained if we assume $M$ has maximum volume growth as in Theorem 1.1.

For $(M, g)$ as in Theorem 1.2 , it is now well known by $[32,34]$ (see also [27]) that (1.3) has a long-time solution $\tilde{g}(t), 0 \leq t<\infty$. If we let $g(x, t)=\mathrm{e}^{-t} \tilde{g}\left(x, \mathrm{e}^{t}\right)$, then we obtain a solution to the normalized KählerRicci flow

$$
\frac{\partial}{\partial t} g_{i \bar{\jmath}}(x, t)=-R_{i \bar{\jmath}}(x, t)-g_{i \bar{\jmath}}(x, t)
$$

for $-\infty<t<\infty$. Hence Theorem 1.2 can be viewed as a uniformization theorem on eternal solutions of (1.4). Motivated by this, we will also prove a uniformization theorem for eternal solutions to the Kähler-Ricci flow (1.3), i.e., a smooth family of complete Kähler metrics $g(t)$ on $M$ satisfying

$$
\frac{\partial}{\partial t} g_{i \bar{\jmath}}(x, t)=-R_{i \bar{\jmath}}(x, t)
$$

for all $t \in(-\infty, \infty)$. We have the following:

Theorem 1.3. Let $(M, g(t))$ be a complete eternal solution to (1.5) such that for all $t, g(t)$ has non-negative holomorphic bisectional curvature which is uniformly bounded on $M$ independent of $t$. Then $M$ is holomorphically covered by $\mathbb{C}^{n}$.

Remark 1.4. Recall that by $[10,22,23,26,28]$, if $M$ is complete noncompact with bounded non-negative bisectional curvature and if the curvature decays faster than quadratic in the average sense, then $M$ is flat. Hence Theorem 1.2 addresses the maximal (quadratic) curvature decay case for nonflat $M$. On the other hand, by the Harnack inequality [4] and the decay estimates in [33, section 6] (see also [27, Corollary 2.1]), it is seen that the average curvature of nonflat $(M, g)$ in Theorem 1.3 cannot decay faster than linearly uniformly at all points and so the decay rate is minimal in some sense by [28]. So Theorem 1.3 addresses the case of minimal (linear) curvature decay.

By comparing (1.4) and (1.5), we may combine Theorems 1.2 and 1.3 as follows:

Theorem 1.5. Let $M^{n}$ be a noncompact complex manifold. Suppose there is a smooth family of complete Kähler metrics $g(t)$ on $M$ such that for 
$\kappa=0$ or $1, g(t)$ satisfies

$$
\frac{\partial}{\partial t} g_{i \bar{\jmath}}(x, t)=-R_{i \bar{\jmath}}(x, t)-\kappa g_{i \bar{\jmath}}(x, t)
$$

for all $t \in(-\infty, \infty)$ such that for every $t, g(t)$ has uniformly bounded non-negative holomorphic bisectional curvature on $M$ independent of $t$. Then $M$ is holomorphically covered by $\mathbb{C}^{n}$.

By [5], if in Theorem 1.5 we also assume that the Ricci curvature is positive and the scalar curvature attains its maximum in spacetime, then $(M, g(t))$ is a gradient Kähler-Ricci soliton of steady type if $\kappa=0$, and of expanding type if $\kappa=1$. If this is the case, then one may use the results on gradient Kähler-Ricci solitons in $[3,8]$ to conclude that $M$ is biholomorphic to $\mathbb{C}^{n}$. Hence Theorem 1.5 can also be considered as a generalization of the results in $[3,8]$.

\section{Local limit solution}

Before we prove the main result, observe that if we take $\pi: \widehat{M} \rightarrow M$ to be the universal holomorphic covering of $M$ in Theorem 1.5 and let $\widehat{g}(t)=$ $\pi^{*}(g(t))$, then $(\widehat{M}, \widehat{g})$ still satisfies the conditions of the theorem. To prove the theorem, it is sufficient to prove that $\widehat{M}$ is biholomorphic to $\mathbb{C}^{n}$. By [6], we may further assume that the Ricci curvature of $\widehat{g}(x, t)$ is positive for all $x$ and $t$. Hence from now on we assume that $M$ in Theorem 1.5 is simply connected and $g(t)$ has positive Ricci curvature for all $t$.

Let $(M, g(t))$ be as above satisfying the conditions of Theorem 1.5. Fix some point $p \in M$, some time sequence $t_{k} \rightarrow \infty$ and consider the sequence $\left(M, g\left(t_{k}+t\right), p\right)$ of long-time solutions to the Kähler-Ricci flow centered at $p$. Suppose the injectivity radius of $g(t)$ at $p$ has a uniform lower bound. Then by Hamilton's compactness [17], this sequence has a convergent subsequence converging to a solution $h(t)$ to the Kähler-Ricci flow on a limit complex manifold $N$. Furthermore, by Cao's classification of limits for Kähler-Ricci flow [5], this limit must either be a steady or expanding gradient Kähler-Ricci soliton, depending on whether $\lambda=0$ or $\lambda=1$. In this section, we show that in the absence of an injectivity radius estimate, we may still have such a soliton limit, but in a local sense. We will consider a certain locally lifted subsequence limit of $\left(M, g\left(t_{k}+t\right), p\right)$ around $p$. Our first goal will be to show that this local limit is also either an expanding or steady gradient Kähler-Ricci soliton in a certain sense (Theorem 2.1). We will then relate this to the local asymptotic behavior of 
$g(t)$ at $p$ in our main Theorem 2.2. In the absence of injectivity radius estimates, Glickenstein [16] constructed a global limit solution from a solution to the Ricci flow as above, allowing for the possibility of Gromov Hausdorff convergence to a limiting metric space of dimension lower than that of $M$. We refer the reader to [16] for details on the construction of this limit and its application, and in particular to $[14,15]$ for applications in three dimensions. Our local limit is just the first step of Glickenstein's construction and in fact depends only on Proposition 2.1 and the simple fact that a lifting of a solution to the Ricci flow is still a solution to the flow. For recent work relating this and, in general, on the existence and classification of limits to the Ricci flow, we refer to the works of Ye [37] and Lott [20].

By $[18,31,34]$, from the time independent bounds on the curvature of $g(t)$, we have corresponding uniform bounds on all covariant derivatives of the curvature by the Kähler-Ricci flow. Hence for $t \geq a$ with $a>-\infty$, we may assume that these bounds on all covariant derivatives of the curvature of $g(t)$ are also time independent. The proof of Proposition 1.2 in [35] then gives (see also [9, Proposition 2.1]):

Proposition 2.1. There exist positive constants $r$ and $C$ such that for each $t \geq-1$ there is a holomorphic map $\Phi_{t}$ from the Euclidean ball $D(r)$ (centered at the origin of $\mathbb{C}^{n}$ with radius $r$ ) to $M$ satisfying the following:

(i) $\Phi_{t}$ is a local biholomorphism from $D(r)$ to $M$;

(ii) $\Phi_{t}(0)=p$;

(iii) $\Phi_{t}^{*}(g(t))(0)=g_{e}$;

(iv) $\frac{1}{C} g_{e} \leq \Phi_{t}^{*}(g(t)) \leq C g_{e}$ in $D(r)$.

(v) for any $0<\alpha<1$, and $k \geq 0$, the standard $C^{k+\alpha}$ norm of $\Phi_{t}^{*}(g(t))$ in $D(r)$ is bounded by a constant $C^{\prime}$ which is independent of $t \geq-1$.

where $g_{e}$ is the standard metric on $\mathbb{C}^{n}$.

Remark 2.2. Proposition 1.2 in [35] only requires the first covariant derivative of the scalar curvature of $g(t)$ to be bounded independent of $t$. In our case, however, we have bounds on all covariant derivatives of the Riemannian curvature tensor independent of $k$. Condition (v) is derived by continuing the argument in [35] or [9].

As in [9], the following proposition is crucial: 
Proposition 2.3. Let $\lambda_{1}(t) \geq \cdots>\lambda_{n}(t)>0$ be the eigenvalues of $R_{i \bar{\jmath}}(p, t)$ relative to $g_{i \bar{\jmath}}(p, t)$.

(i) For any $\tau>0$,

$$
\phi=\frac{\operatorname{det}\left(R_{i \bar{\jmath}}(p, t)+\tau g_{i j}\right)}{\operatorname{det}\left(g_{i \bar{\jmath}}(p, t)\right)}
$$

is nondecreasing in $t$.

(ii) There is a constant $C>0$ such that $\lambda_{n}(t) \geq C$ for all $t \geq 0$.

(iii) For $1 \leq i \leq n$, the limit $\lim _{t \rightarrow \infty} \lambda_{i}(t)$ exists.

(iv) Let $\mu_{1}>\cdots>\mu_{l}>0$ be the distinct limits in (iii) and let $\rho>0$ be such that the intervals $\left[\mu_{k}-\rho, \mu_{k}+\rho\right]$ for $1 \leq k \leq l$ are disjoint. For any $t$, let $E_{k}(t)$ be the sum of the eigenspaces corresponding to the eigenvalues $\lambda_{i}(t)$ such that $\lambda_{i}(t) \in\left(\mu_{k}-\rho, \mu_{k}+\rho\right)$. Let $P_{k}(t)$ be the orthogonal projection (with respect to $g(t)$ ) onto $E_{k}(t)$. Then there exists $T>0$ such that if $t>T$ and if $w \in T_{p}^{(1,0)}(M),\left|P_{k}(t)(w)\right|_{t}$ is continuous in $t$, where $|\cdot|_{t}$ is the length measured with respect to the metric $g(p, t)$.

Proof. The proof is identical to the proof of Proposition 3.1 in [9] for $\kappa=1$. Suppose $\kappa=0$. By Theorem 2.3 in [5], if

$$
Z_{i \bar{\jmath}}=\frac{\partial R_{i \bar{\jmath}}}{\partial t}+g^{k \bar{l}} R_{i \bar{l}} R_{k \bar{j}}
$$

then

$$
Z_{i \bar{\jmath}} w^{i} w^{\bar{j}} \geq 0
$$

for any $w \in T^{(1,0)}(M)$. Let $p_{i \bar{\jmath}}=R_{i \bar{\jmath}}+\tau g_{i \bar{\jmath}}$ and denote its inverse by $\left(p^{i \bar{\jmath}}\right)$. We have

$$
\begin{aligned}
\frac{\partial}{\partial t} \log \phi & =p^{i \bar{\jmath}} \frac{\partial}{\partial t} p_{i \bar{\jmath}}-g^{i \bar{\jmath}} \frac{\partial}{\partial t} g_{i \bar{\jmath}} \\
& =p^{i \bar{\jmath}}\left(\frac{\partial}{\partial t} R_{i \bar{\jmath}}-\tau R_{i \bar{\jmath}}\right)+g^{i \bar{\jmath}} R_{i \bar{\jmath}} \\
& \geq p^{i \bar{\jmath}}\left(-g^{k \bar{l}} R_{i \bar{l}} R_{k \bar{j}}-\tau R_{i \bar{\jmath}}\right)+g^{i \bar{\jmath}} R_{i \bar{\jmath}} \\
& =p^{i \bar{\jmath}}\left(-g^{k \bar{l}} R_{i \bar{l}} R_{k \bar{j}}-\tau p_{i \bar{\jmath}}\right)+\tau^{2} p^{i \bar{\jmath}} g_{i \bar{\jmath}}+g^{i \bar{\jmath}} R_{i \bar{\jmath}}
\end{aligned}
$$


Now at the point $(p, t)$, we choose a unitary basis such that $g_{i \bar{\jmath}}=\delta_{i j}$ and $R_{i \bar{\jmath}}=\lambda_{i} \delta_{i j}$. Then $p_{i \bar{\jmath}}=\left(\lambda_{i}+\tau\right) \delta_{i j}$ and $p^{i \bar{\jmath}}=\left(\lambda_{i}+\tau\right)^{-1} \delta_{i j}$. Hence, we have

$$
\begin{aligned}
\frac{\partial}{\partial t} \log \phi & \geq-\sum_{i=1}^{n} \frac{\lambda_{i}^{2}}{\lambda_{i}+\tau}-\tau n+\sum_{i=1}^{n} \frac{\tau^{2}}{\lambda_{i}+\tau}+\sum_{i=1}^{n} \lambda_{i} \\
& =\sum_{i=1}^{n}\left(\frac{-\lambda_{i}^{2}}{\lambda_{i}+\tau}-\tau+\frac{\tau^{2}}{\lambda_{i}+\tau}+\lambda_{i}\right) \\
& =0 .
\end{aligned}
$$

From this (i) follows. The proof of (ii)-(iv) is similar to the proof of (ii)-(iv) in Proposition 3.1 of [9].

For each $k$, consider the lifted family of metrics $g_{k}(t):=\Phi_{t_{k}}^{*} g\left(t_{k}+t\right)$ on $D(r)$ for $t \in[-1, \infty)$, say. Then it is easy to see that $g_{k}(t)$ solves the Kähler-Ricci flow (1.6) on $D(r)$. Then by Proposition 2.1 and the KählerRicci flow it follows that some subsequence of $g_{k}(t)$ converges to a smooth limit family $h(t)$, uniformly on compact subsets of $D(r) \times(-1, \infty)$. It is easy to see that these are Kähler metrics on $D$ for all $t$ and that $h(t)$ solves (1.6). Moreover, by Proposition 2.3, the eigenvalues of the Ricci tensor $R_{i \bar{\jmath}}^{h}(t)$ of $h(t)$ at the origin are equal to $\lim _{s \rightarrow \infty} \lambda_{i}(s)$ for any $t \in[0, \infty)$. Therefore, $\mu_{1}>\mu_{2}>\cdots>\mu_{l}>0$ are distinct eigenvalues of $R_{i \bar{\jmath}}^{h}(t)$ at the origin. By the uniform bounds on the covariant derivatives of the curvature tensor of $h(t)$ in $D(r) \times(-1, \infty)$, and by Proposition 2.3 , we may have the following inequality on $D(r)$, for $t \geq-1 / 2$, and by choosing a smaller $r$ if necessary:

$$
R_{i \bar{\jmath}}^{h} \geq C h_{i \bar{\jmath}}
$$

Theorem 2.4. Let $R c_{i \bar{\jmath}}^{h}(t)$ be the Ricci tensor of the metric $h_{i \bar{\jmath}}(t)$ on $D(r)$.

(i) For each $t \in[0, \infty)$ we have

$$
R c_{i \bar{\jmath}}^{h}(t)+\kappa h_{i \bar{\jmath}}(t)=f_{i \bar{\jmath}}(t)
$$

for some smooth real-valued function $f(t)$ on $D(r)$ such that $f_{i j}(t)=0$ and the gradient of $f(t)$ in $h(t)$ is zero at the origin.

(ii) Let $\mu_{1}>\mu_{2}>\cdots>\mu_{l}>0$ be as above. For $1 \leq i \leq l$, let $E_{i}$ be the eigenspace corresponding to $\mu_{i}$ of $\operatorname{Ric}^{h}(0,0)$ at $t=0$ at the origin with respect to $h(0)$. Then $E_{i}$ is also the eigenspace corresponding to $\mu_{i}$ of $\operatorname{Ric}^{h}(0, t)$ for all $t \geq 0$ at the origin with respect to $h(t), 1 \leq i \leq l$. 
To prove the theorem, we first prove a lemma which is a direct modification of the results in [5]. In the case of $\kappa=1$, it will be more convenient to consider the transformed metric $\tilde{h}(t)=t h(\log t)$ which solves (1.6) on $D(r) \times\left[e^{-1}, \infty\right)$ with $\kappa=0$. It is clear that $\tilde{h}(t)$ is the limit of the transformed sequence $\tilde{g}_{k}(t):=t g_{k}(\log t)$ uniform on compact sets of $D(r) \times\left[e^{-1}, \infty\right)$ which also satisfy $(1.6)$ with $\kappa=0$.

Let $Z_{i \bar{\jmath}}$ and $Z_{i \bar{\jmath}}^{k}$ be the Harnack quadratic tensors corresponding to $\tilde{h}(t)$ and $\tilde{g}_{k}(t)$, respectively, as defined in Theorem 2.1 in [5]. Namely for any holomorphic vector $\left(V^{i}\right)$ at a point $q \in D(r)$,

$$
Z_{i \bar{\jmath}}=\frac{\partial}{\partial t} R_{i \bar{\jmath}}^{\tilde{h}}+\tilde{h}^{l \bar{k}} R_{i \bar{k}}^{\tilde{h}} R_{l \bar{\jmath}}^{\tilde{h}}+R_{i \bar{\jmath}, k}^{\tilde{h}} V_{\bar{k}}+R_{i \bar{\jmath}, \bar{k}}^{\tilde{h}} V_{k}+R_{i \bar{\jmath} k \bar{l}}^{\tilde{h}} V_{\bar{k}} V_{l}+\frac{1}{t} R_{i \bar{\jmath}}^{\tilde{h}}
$$

and $Z_{i \bar{\jmath}}^{k}$ is defined similarly. Denote the trace $\tilde{h}^{i \bar{\jmath}} Z_{i \bar{\jmath}}$ of $Z_{i \bar{\jmath}}$ by $Z$. Note that $Z$ is a smooth function defined on the holomorphic tangent bundle $T^{(1,0)}(D(r))$.

In case $\kappa=0$, then let $Q_{i \bar{\jmath}}$ and $Q_{i \bar{\jmath}}^{k}$ be the Harnack quadratic tensors corresponding to $h(t)$ and $g_{k}(t)$, respectively, as defined in Theorem 2.3 in [5]. Namely for any holomorphic vector $\left(V^{i}\right)$ at a point $x \in D(r)$,

$$
Q_{i \bar{\jmath}}=\frac{\partial}{\partial t} R_{i \bar{\jmath}}^{h}+h^{l \bar{k}} R_{i \bar{k}}^{h} R_{l \bar{j}}^{h}+R_{i \bar{\jmath}, k}^{h} V_{\bar{k}}+R_{i \bar{\jmath}, \bar{k}}^{h} V_{k}+R_{i \bar{j} k \bar{l}}^{h} V_{\bar{k}} V_{l}
$$

and $Q_{i \bar{\jmath}}^{k}$ is defined similarly. Denote the trace $h^{i \bar{\jmath}} Q_{i \bar{\jmath}}$ of $Q_{i \bar{\jmath}}$ by $Q$.

\section{Lemma 2.5.}

(i) For any holomorphic vector $V \in T^{(1,0)}(D(r)), Z_{i \bar{\jmath}}$ is a non-negative quadratic form. Moreover, if $Z$ is positive at some point $x_{0} \in D(r)$ for all $V \in T_{x_{0}}^{(1,0)}(D(r))$ at $t=t_{0}$, then $Z$ is positive for all $t>t_{0}$ and for $V \in T^{(1,0)}(D(r))$.

(ii) For any holomorphic vector $V \in T^{(1,0)}(D(r)), Q_{i \bar{\jmath}}^{h}$ is a non-negative quadratic form. Moreover, if $Q^{h}$ is positive at some point $x_{0} \in D(r)$ for all $V \in T_{x_{0}}^{(1,0)}(D(r))$ at $t=t_{0}$, then $Q^{h}$ is positive for all $t>t_{0}$ and for $V \in T^{(1,0)}(D(r))$.

Proof. For any holomorphic vector $W, Z_{i \bar{j}}^{k} W^{i} W^{\bar{j}} \geq 0$ for all $k$ by Theorem 2.1 in [5]. Since $Z_{i \bar{\jmath}}$ is the limit of the $Z_{i \bar{\jmath}}^{k}$ 's on $D(r)$ for all $t, Z_{i \bar{\jmath}} W^{i} W^{\bar{j}} \geq 0$. This proves the first statement of (i). The first statement of (ii) can be proved similarly.

To prove the second statement in (i), assume there is some $x_{0} \in D(r)$ and $t_{0} \geq e^{-1}$ so that $Z>0$ for all $V \in T_{x_{0}}^{(1,0)}(D(r))$. Given any $T>t_{0}$, 
we note that for $C>0$ there exists some $K>0$ such that given any point $(x, t) \in D(r) \times\left[t_{0}, T\right]$ and $V \in T_{x}^{(1,0)}(D(r))$, with Euclidean length $\|V\|>$ $K$, we must have

$$
Z>C
$$

at $(x, t)$ and $V$. This follows from (2.6), (2.5) and the fact that the curvature tensor of $\tilde{h}(t)$ and its covariant derivatives in time and space are uniformly bounded on $D \times\left[t_{0}, T\right]$ by constants independent of space and time by our estimates on the $\tilde{g}_{k}^{\prime} s$. Hence there exist a neighborhood $U$ of $x_{0}$ and $\epsilon>0$ such that $Z \geq \epsilon$ for all holomorphic vector $V$ at $x \in U$ at $t=t_{0}$.

Choose a smooth function $F$ on $D(r)$ such that $F\left(x_{0}\right)>0, F$ is zero outside a small neighborhood of $x_{0}$ and $Z-\frac{F}{t_{0}^{2}} \geq 0$ for all $V$ in $T^{(1,0)}(D(r))$ at $t=t_{0}$.

Let $F$ evolve by the heat equation on $D \times\left[t_{0}, T\right]$ with the following initial and boundary conditions:

$$
\begin{aligned}
\frac{\partial}{\partial t} F & =\Delta_{t} F \quad \text { in } \quad D \times\left[t_{0}, T\right] \\
F & =0 \quad \text { on } \quad \partial D \times\left[t_{0}, T\right] \\
F\left(x, t_{0}\right) & =F(x)
\end{aligned}
$$

where $\Delta_{t}$ is the Laplacian relative to $\tilde{h}(t) . \quad F$ is then strictly positive in $D \times\left(t_{0}, T\right]$ by the strong maximum principle [29, Theorem 5 , Chapter 3] We will show that $\tilde{Z}:=Z-\frac{F}{t^{2}}$ is also non-negative for all $V$ and $(x, t) \in$ $D \times\left(t_{0}, T\right]$. Without loss of generality we may assume $\tilde{h}$ is smooth up to the boundary of $D(r)$. Let $\tilde{Z}$ assume its minimum over all $(x, t) \in \bar{D}(r) \times$ $\left[t_{0}, T\right]$ and $V$, at some point $\left(x, t_{1}\right)$ and some vector $V_{0}$. This minimum exists by compactness and by (2.8). Now assume this minimum is negative. Then by the initial condition of $F$, we must have $t_{1}>t_{0}$. Also, by the non-negativity of $Z$ and the zero Dirichlet boundary condition on $F$, we must have $x$ strictly inside $D(r)$. As in [5], we may then extend $V_{0}$ locally around $\left(x, t_{1}\right)$ in space-time such that $(3.2)$ in [5] holds. At $\left(x, t_{1}\right)$ we then have

$$
\begin{aligned}
\left(\frac{\partial}{\partial t}-\Delta_{t}\right) \tilde{Z} & =\left(\frac{\partial}{\partial t}-\Delta_{t}\right) Z+2 \frac{F}{t^{3}} \\
& =Z_{i \bar{\jmath}} R_{j \bar{\imath}}^{\tilde{h}}-2 \frac{Z}{t}+2 \frac{F}{t^{3}}
\end{aligned}
$$




$$
\begin{aligned}
& =Z_{i \bar{\jmath}} R_{j \bar{\imath}}^{\tilde{h}}-2 \frac{\tilde{Z}}{t} \\
& >0 .
\end{aligned}
$$

But this contradicts the fact that $\tilde{Z}$ is minimal at $\left(x, t_{1}\right)$ and $V_{0}$. Thus $\tilde{Z}$ is non-negative as claimed, which completes the proof of (i) of the lemma.

We now consider the second statement of (ii). Assume there is some $x_{0} \in D(r)$ and $t_{0} \geq 0$ such that $Q$ is positive at $\left(x_{0}, t_{0}\right)$ for all $V$. As before, for any $T>t_{0}$ we observe that given any $C>0$, there exists some $K>0$ such that given any point $(x, t) \in D(r) \times\left[t_{0}, T\right]$ and $V \in T_{x}^{(1,0)}(D(r))$ with Euclidean length $\|V\|>K$, we must have $Z>C$ at $(x, t)$ and $V$.

Hence we can choose a smooth function $F$ on $D$ such that $F\left(x_{0}\right)>0, F$ is zero outside a small neighborhood of $x_{0}$ and $Q-F \geq 0$ for all $V$ everywhere on $D(r)$ at $t=t_{0}$. Let $F$ evolve by heat equation on $D \times\left[t_{0}, T\right]$ with the following initial and boundary conditions:

$$
\begin{aligned}
\frac{\partial}{\partial t} F & =\Delta_{t} F \quad \text { in } \quad D(r) \times\left[t_{0}, t\right] \\
F & =0 \quad \text { on } \quad \partial D(r) \times\left[t_{0}, T\right] \\
F\left(x, t_{0}\right) & =F(x)
\end{aligned}
$$

where $\Delta_{t}$ is the Laplacian relative to $h(t) . \quad F$ is then strictly positive in $D \times\left(t_{0}, T\right]$ by the strong maximum principle. Now given any $\epsilon>0$, we will show that $\tilde{Q}:=Q-F+\epsilon e^{t}$ is non-negative for all $V$ and $(x, t) \in$ $D(r) \times\left(t_{0}, T\right]$. Letting $\epsilon$ approach zero, this will prove that $Q-F$ is nonnegative for all $V$ and $(x, t) \in D(r) \times\left(t_{0}, T\right]$, thus proving the lemma.

Let $\tilde{Q}$ assume its minimum over all $(x, t) \in D(r) \times\left[t_{0}, T\right]$ and $V$, at some point $(x, t)$ and some vector $V_{0}$. Now assume this minimum is negative. Then by our initial condition of $F$, we must have $t>t_{0}$. Also, by the non-negativity of $Q$ and the zero Dirichlet boundary condition on $F$, we must have $x$ strictly inside $D(r)$. We may then extend $V_{0}$ locally around $(x, t)$ in space-time such that $(3.1)$ in [5] holds. At $(x, t)$ we then have

$$
\begin{aligned}
\left(\frac{\partial}{\partial t}-\Delta_{t}\right) \tilde{Q} & =\left(\frac{\partial}{\partial t}-\Delta_{t}\right) Q+\epsilon e^{t} \\
& =Q_{i \bar{\jmath}} R_{j \bar{i}}^{\tilde{h}}+\epsilon e^{t} \\
& >0 .
\end{aligned}
$$


But this contradicts the fact that $\tilde{Q}$ is minimal at $(x, t)$ and $V_{0}$. Thus $\tilde{Q}$ is non-negative as claimed, which completes the proof of (ii).

Proof of Theorem 2.4. (i): We begin with the case where $\kappa=1$ in Theorem 2.4. With the same notations as in the Lemma 2.5, by Proposition 2.3 and the arguments following (3.7) in [9], we see that

$$
t R^{\tilde{h}}(0, t)
$$

is constant for all $t \in\left[e^{-1}, \infty\right)$ where $R_{\tilde{h}}^{\tilde{h}}$ is the scalar curvature of $\tilde{h}$. Thus at the space time point $(0, t)$ we have $R^{\tilde{h}}+t \frac{\partial}{\partial t} R^{\tilde{h}}=0$. Applying Lemma 2.1 and following the exact argument in the proof of Theorem 4.2 in [5], we conclude that for each $t \in\left[e^{-1}, \infty\right)$ there is a smooth real-valued function $\tilde{f}(t)$ on $D(r)$ such that the gradient of $\tilde{f}(t)$ is holomorphic and is zero at the origin. Moreover, we have

$$
R c_{i \bar{\jmath}}^{\tilde{h}}=\tilde{f}_{i \bar{\jmath}}+\frac{1}{t} \tilde{h}_{i \bar{\jmath}}
$$

Transforming $\tilde{h}$ back to $h$, it is easy to see that (i) in Theorem 2.4 is true for $\kappa=1$.

We now consider the case of $\kappa=0$. By Proposition 2.3 we have $R^{h}(0, t)$ is constant for $t \in[0, \infty)$, and in particular, $\frac{\partial}{\partial t} R^{h}=0$ at the space time point $(0, t)$. Applying Lemma 2.5 and following the exact argument in the proof of Theorem 4.1 in [5], we conclude that for each $t \in[0, \infty)$ there is a smooth real-valued function $f(t)$ on $D(r)$ such that the gradient of $f(t)$ is holomorphic and is zero at the origin. Moreover, we have

$$
R c_{i \bar{\jmath}}^{h}=f_{i \bar{\jmath}} .
$$

This completes the proof of (i) in Theorem 2.4.

(ii): Let $\lambda_{1} \geq \cdots \geq \lambda_{n}>0$ be the eigenvalues of $R c^{h}$ at 0 . Note that they are independent of $t$. Suppose $v \in T_{0}^{1,0}(D(r))$ is not an eigenvector for $R c^{h}(0,0)$ for $\lambda_{k}$. We will show that $v$ cannot be an eigenvector for $R c^{h}(0, t)$ for all $t \in(0, \infty)$ for $\lambda_{k}$. It is sufficient to prove that the quantity

$$
\begin{aligned}
F(t):= & \left|R c^{h}(0, t)(v, \cdot)-\lambda_{k} h(0, t)(v, \cdot)\right|_{h(t)}^{2} \\
= & h^{j \bar{k}}(0, t) \overline{\left(R_{i \bar{\jmath}}^{h}(0, t)-\lambda_{k} h_{i \bar{\jmath}}(0, t)\right) v^{i}} \\
& \cdot\left(R_{l \bar{k}}^{h}(0, t)-\lambda_{k} h_{l \bar{k}}(0, t)\right) v^{l}
\end{aligned}
$$

can never be zero since this is zero at $t$ if and only if $v$ is an eigenvector for $R c^{h}(0, t)$ with eigenvalue $\lambda_{k}$. Let $t_{0} \in[0, \infty)$ be arbitrary. Choose a 
holomorphic coordinate in $D(r)$ such that at $0 \in D(r)$ we have $h_{i \bar{\jmath}}\left(t_{0}\right)=\delta_{i \bar{\jmath}}$ and $R_{i \bar{\jmath}}^{h}\left(t_{0}\right)=\lambda_{i} \delta_{i \bar{\jmath}}$. Let $f(t)$ be as in Lemma 2.5. It is not hard to show that we may choose some $1>\delta>0$ such that starting at any point $p \in D(\delta r)$ we may flow along $-\frac{1}{2} \nabla f\left(t_{0}\right)$ for $t \in[0,1]$ while staying inside $D(r)$ and let $\varphi_{t}$ be the local biholomorphism determined by the flow. Note that the origin is a fixed point of the flow because $\nabla f(t)=0$ at the origin. Let $g(t)=\varphi_{t}^{*}\left(h\left(t_{0}\right)\right)$ be the soliton metric on $D(\delta r) \times[0,1)$ with initial condition $g(0)=h\left(t_{0}\right)$. Then at $0 \in D(\delta r)$, in the above coordinates, we have $g_{i \bar{\jmath}}(t)=e^{-\left(\lambda_{i}+\kappa\right) t} \delta_{i \bar{\jmath}}$ and $R_{i \bar{\jmath}}^{g}(t)=\lambda_{i} e^{-\left(\lambda_{i}+\kappa\right) t} \delta_{i \bar{\jmath}}$. For any $k$ we then have

$$
\begin{aligned}
G(t) & :=\left|R c^{g}(0, t)(v, \cdot)-\lambda_{k} g(0, t)(v, \cdot)\right|_{g(t)}^{2} \\
& =\sum_{i \neq k}\left(\lambda_{i}-\lambda_{k}\right)^{2}\left|v^{i}\right|^{2} e^{-\left(\lambda_{i}+\kappa\right) t}
\end{aligned}
$$

and thus

$$
\begin{aligned}
G^{\prime}(0) & =\sum_{i \neq k}-\left(\lambda_{i}+\kappa\right)\left(\lambda_{i}-\lambda_{k}\right)^{2}\left|v^{i}\right|^{2} \\
& \geq-\left(\lambda_{1}+\kappa\right) G(0) .
\end{aligned}
$$

From the above equation, the fact that $g(0)=h\left(t_{0}\right)$ on $D(\delta r)$, and the fact that both $g(t)$ and $h\left(t_{0}+t\right)$ ) solve (1.6) on $D(\delta r) \times[0,1)$, it follows that $G(0)=F\left(t_{0}\right)$ and $G^{\prime}(0)=F^{\prime}\left(t_{0}\right)$. Hence for any choice of $k$ we have $F^{\prime}\left(t_{0}\right) \geq$ $-\left(\lambda_{1}+\kappa\right) F\left(t_{0}\right)$. But $t_{0} \in[0, \infty)$ is arbitrary. Thus for any $k$ we have $F^{\prime}(t) \geq$ $-\left(\lambda_{1}+\kappa\right) F(t)$ for all $t \in[0, \infty)$. It is now easy to see that if $F(0) \neq 0$, then $F(t)$ cannot be zero for any $t$. This completes the proof of our claim.

From the claim, we conclude that if $v$ is an eigenvector of $h(t)$ for $t>0$ with eigenvalue $\lambda_{k}$, then $v$ must be in $E_{k}$. Since the multiplicity of each eigenvalue $\mu_{k}$ is constant in $t$, from this it is easy to see the theorem is true.

Now given $(M, g(t))$ as in the beginning of the section, we denote the eigenvalues of $R c(p, t)$ by $\lambda_{i}(t)$ for $i=1, \ldots, n$ as before, and we let $\mu_{k}, E_{k}(t)$ and $P_{k}(t)$ for $k=1, \ldots, l$ be as in Proposition 2.3. We let $n_{m}$ for $m=1$, $1, \ldots, l+1$ with $n_{1}=0$ and $n_{l+1}=n$ be such that $\lambda_{i} \in\left(\mu_{m}-\rho, \mu_{m}+\rho\right)$ for all $n_{m}<i \leq n_{m+1}$ and $m=1, \ldots, l$ and $t$ sufficiently large such that the intervals $\left[\mu_{m}-\rho, \mu_{m}+\rho\right]$ are disjoint as in Proposition 2.3 part (iv). For any nonzero vector $v \in T_{p}^{1,0}(M)$, let $v(t)=v /|v|_{t}$, where $|v|_{t}$ is the length of $v$ with respect to $g(t)$ and $v_{i}(t)=P_{i}(t) v(t)$.

We now show that Theorem 4.1 in [9] is also true for $(M, g(t))$ in our case; that $R c(p, t)$ can be "diagonalized" simultaneously near infinity and that $g(t)$ is "Lyapunov regular", to borrow a notion from dynamical systems (see [1]). 
Theorem 2.6. Let $(M, g(t))$ be as described in the beginning of the section. Then $V=T_{p}^{(1,0)}(M)$ can be decomposed orthogonally with respect to $g(0)$ as $V_{1} \oplus \cdots \oplus V_{l}$ so that the following are true:

(i) If $v$ is a nonzero vector in $V_{i}$ for some $1 \leq i \leq l$, then $\lim _{t \rightarrow \infty}\left|v_{i}(t)\right|=$ 1 and thus $\lim _{t \rightarrow \infty} R c(v(t), \bar{v}(t))=\mu_{i}$ and

$$
\lim _{t \rightarrow \infty} \frac{1}{t} \log \frac{|v|_{t}^{2}}{|v|_{0}^{2}}=-\mu_{i}-\kappa
$$

Moreover, the convergence are uniform over all $v \in V_{i} \backslash\{0\}$.

(ii) For $1 \leq i, j \leq l$ and for nonzero vectors $v \in V_{i}$ and $w \in V_{j}$ where $i \neq j$, $\lim _{t \rightarrow \infty}\langle v(t), w(t)\rangle_{t}=0$ and the convergence is uniform over all such nonzero vectors $v, w$.

(iii) $\operatorname{dim}_{\mathbb{C}}\left(V_{i}\right)=n_{i+1}-n_{i}$ for each $i$.

$$
\sum_{i=1}^{l}\left(-\mu_{i}-\kappa\right) \operatorname{dim}_{\mathbb{C}} V_{i}=\lim _{t \rightarrow \infty} \frac{1}{t} \log \frac{\operatorname{det}\left(g_{i \bar{j}}(t)\right)}{\operatorname{det}\left(g_{i \bar{j}}(0)\right.}
$$

Proof. Let $t_{k} \rightarrow \infty$ and construct $g_{k}$ with limit $h(t)$ as in Theorem 2.4. Observe that since $h(t)$ is a smooth limit of the $g_{k}(t)^{\prime} s$ on $D(r) \times[0, \infty)$, the analog of Lemma 3.2 in [9] is true in our case. Using this and (ii) in Theorem 2.4, we may prove the theorem by contradiction exactly as in the proof of Theorem 4.1 in [9].

\section{Transition maps}

Let $(M, g(t))$ be as in Theorem 1.5 and let $p \in M$ be fixed. In addition, we will assume that $R_{i \bar{\jmath}} \geq a^{\prime} g_{i \bar{\jmath}}$ for some $a^{\prime}>0$ at $p$ and $t=0$. Then by Proposition 2.3, there exists $a>0$ such that

$$
R_{i \bar{\jmath}} \geq a g_{i \bar{\jmath}}
$$

at $p$ for all $t \geq 0$. Since the covariant derivatives Riemannian curvature tensor $R m_{t}$ of $g(t)$ is uniformly bounded, we can conclude that by choosing a possibly smaller $a>0$, that $(3.1)$ is still true in $B_{t}(p, R)$ for some $R>0$ independent of $t$, where $B_{t}(p, R)$ is the geodesic ball of radius $R$ with center at $p$ with respect to the metric $g(t)$. Since $B_{t_{1}}(p, R) \subset B_{t_{2}}(p, R)$ for $t_{2} \geq t_{1}$ 
as $g(t)$ is shrinking, we have

$$
L_{t_{2}}(\gamma) \leq e^{-\frac{a}{2}\left(t_{2}-t_{1}\right)} L_{t_{1}}(\gamma)
$$

for any curve in $B_{t_{1}}(p, R)$. Here $L_{t}$ denotes the length function with respect to $g(t)$.

Recall that by Proposition 2.1, there exist $r>0$ and $C>0$ independent of $t \geq 0$ and a holomorphic maps $\Phi_{t}: D(r) \rightarrow M$ with the following properties:

(i) $\Phi_{t}$ is a local biholomorphism from $D(r)$ onto its image;

(ii) $\Phi_{t}(0)=p$;

(iii) $\Phi_{t}^{*}(g(t))(0)=g_{e}$;

(iv) $\frac{1}{C} g_{e} \leq \Phi_{t}^{*}(g(t)) \leq C g_{e}$ in $D(r)$.

where $g_{e}$ is the standard metric on $\mathbb{C}^{n}$. Let $T>0$ and denote $\Phi_{i T}$ simply by $\Phi_{i}$. In this section, we want to construct injective holomorphic maps $F_{i}$ from $D(\rho)$ to $D(\rho)$ for some $\rho$ such that $\Phi_{i}=\Phi_{i+1} \circ F_{i+1}$. We should emphasize that $\Phi_{i}$ may not be a covering map.

In this section, we always assume that $t \geq 0$. By property (iv) and reducing $r$ if necessary, we may assume that $\Phi_{t}(D(r)) \subset B_{t}(p, R)$, where $R>0$ is such that (3.2) is true. In fact, we have the following:

Lemma 3.1. For any $0<\rho<r$, there exists $R_{1}>0$ independent of $t$ such that

$$
B_{t}\left(p, \frac{1}{R_{1}}\right) \subset \Phi_{t}(D(\rho)) \subset B_{t}\left(p, R_{1}\right)
$$

Proof. By (iv) above, it is easy to see that

$$
\Phi_{t}(D(\rho)) \subset B_{t}\left(p, C_{1}\right)
$$

for some $C_{1}>0$ independent of $t$, where $B_{t}(p, r)$ is the geodesic ball with radius $r$ centered at $p$ with respect to $g(t)$. On the other hand, $\widehat{B}_{t}\left(0, C_{2}\right) \subset$ $D(\rho)$ for some $C_{2}>0$ independent of $t$, where $\widehat{B}_{t}\left(0, C_{2}\right)$ is the geodesic ball with radius $C_{2}$ centered at 0 with respect to $\Phi_{t}^{*}(g(t))$. Hence

$$
\Phi_{t}(D(\rho)) \supset \Phi_{t}\left(\widehat{B}_{t}\left(0, C_{2}\right)\right) \supset B_{t}\left(p, C_{2}\right) .
$$

From this it is easy to see the lemma follows. 
Lemma 3.2. For any $0<\rho \leq r$, where $r$ is as in (i)-(iv), there exists $\rho_{1}>0$ independent of $t$, satisfying the following for any $t$ : Let $\gamma$ be a smooth curve in $M$ with $\gamma(0)=q$ such that $q \in B_{t}\left(p, \rho_{1}\right)$ and $L_{t}(\gamma)<\rho_{1}$. Then $\Phi_{t}(z)=q$ for some $z \in D\left(\frac{\rho}{8}\right)$, and for all such $z$ there is a unique lift $\widetilde{\gamma}$ of $\gamma$ by $\Phi_{t}$ so that $\widetilde{\gamma}(0)=z$ and $\widetilde{\gamma} \subset D\left(\frac{\rho}{2}\right)$.

Proof. Let $\rho_{1}>0$ be determined later. Let $q \in B_{t}\left(p, \rho_{1}\right)$ and let $\gamma(s), 0 \leq$ $s \leq \rho_{1}$ be a curve from $q$ parametrized by arc-length with respect to $g(t)$. Suppose $z \in D\left(\frac{\rho}{8}\right)$ with $\Phi_{t}(z)=q$

Since $\Phi_{t}$ is a local biholomorphism, there exists $s_{0}>0$ and a curve $\widetilde{\gamma}$ from $z$ with $\widetilde{\gamma} \subset D\left(\frac{1}{8} \rho\right)$ such that $\Phi_{t} \circ \widetilde{\gamma}=\gamma$ on $\left[0, s_{0}\right]$. Let $A$ be the set of $s$, such that $\gamma$ has a lift $\widetilde{\gamma}$ in $D\left(\frac{1}{2} \rho\right)$ on $[0, s]$ with $\widetilde{\gamma}(0)=z$. Since $\Phi_{t}$ is a local biholomorphism, $A$ is open in $\left[0, \rho_{1}\right]$. Suppose $s_{k} \rightarrow s$ and $s_{k} \in A$. By (iv), there is a constant $C>0$ which is independent of $t$ such that

$$
C^{-1} L_{e}\left(\left.\widetilde{\gamma}\right|_{\left[0, s_{k}\right]}\right) \leq L_{t}\left(\left.\gamma\right|_{\left[0, s_{k}\right]}\right) \leq \rho_{1},
$$

where $L_{e}$ is the length with respect to $g_{e}$. Hence

$$
L_{e}\left(\left.\widetilde{\gamma}\right|_{\left[0, s_{k}\right]}\right) \leq C \rho_{1} \leq \frac{1}{4} \rho
$$

if $\rho_{1}<\frac{1}{4 C} \rho$. Note that since $\widetilde{\gamma}(0)=z \in D\left(\frac{1}{8} \rho\right)$, we may assume that $\widetilde{\gamma}\left(s_{k}\right) \rightarrow$ $z_{1}$ for some $z_{1} \in \overline{D\left(\frac{3}{8} \rho\right)}$. From this it is easy to see that $\gamma$ can be lifted up to $s$ while staying in $D\left(\frac{1}{2} \rho\right)$. Hence $A$ is also closed. Since $\Phi_{t}$ is a local biholomorphism, the lifting must be unique. In particular, by choosing a smaller $\rho_{1}$ which is independent of $t$, we conclude that every minimal geodesic from $p$ with length less than $\rho_{1}$ can be lifted to a curve in $D\left(\frac{1}{8} \rho\right)$. Hence for all $q \in B_{t}\left(p, \rho_{1}\right)$, there is a point $z \in D\left(\frac{1}{8} \rho\right)$ such that $\Phi_{t}(z)=q$. This completes the proof of the lemma.

Lemma 3.3. Fix $t \geq 0$. Let $0<\rho \leq r$ be given and let $\rho_{1}$ be as in Lemma 3.2. Given any $\epsilon>0$, there exists $\delta>0$ satisfying the following properties: Let $\gamma(\tau), \beta(\tau), \tau \in[0,1]$ be smooth curves from $q \in B_{t}\left(p, \rho_{1}\right)$ with length less than $\rho_{1}$ and let $z_{0} \in D\left(\frac{1}{8} \rho\right)$ with $\Phi_{t}\left(z_{0}\right)=q$. Let $\widetilde{\gamma}, \widetilde{\beta}$ be the liftings from $z_{0}$ of $\gamma$ and $\beta$ as described in Lemma 3.2. Suppose $d_{t}(\gamma(\tau), \beta(\tau))<\delta$ for all $\tau \in[0,1]$, then $d_{e}(\widetilde{\gamma}(1), \widetilde{\beta}(1))<\epsilon$. Here $d_{t}$ is the distance in $g(t)$ and $d_{e}$ is the Euclidean distance.

Proof. Since $\Phi_{t}$ is a local biholomorphism, there is $\sigma>0$ such that $\Phi_{t}$ is a biholomorphism onto its image when restricted on $D(z, \sigma)$ for all $z \in \overline{D\left(\frac{1}{2} \rho\right)}$, where $D(z, \sigma)$ is the Euclidean ball with center at $z$ and radius $\sigma$. 
Let $q, z_{0}, \gamma, \beta, \widetilde{\gamma}$ and $\widetilde{\beta}$ as in the lemma. Since $\widetilde{\gamma} \subset D\left(\frac{1}{2} \rho\right)$, by property (iv) of $\Phi_{t}$, there exists $C_{1}>0$ such that

$$
\Phi_{t}(D(\widetilde{\gamma}(\tau)), \sigma) \supset B_{t}\left(\gamma(\tau), C_{1}^{-1} \sigma\right)
$$

and

$$
d_{e}(\widetilde{\gamma}(\tau), z) \leq C_{1} d_{t}\left(\gamma(\tau), \Phi_{t}(z)\right)
$$

for all $z \in D(\widetilde{\gamma}(\tau), \sigma)$ with $\Phi_{t}(z) \in B_{t}\left(\gamma(\tau), C_{1}^{-1} \sigma\right)$. Note that $C_{1}$ is independent of the curves $\gamma$ and $\beta$.

Given $0<\epsilon<\sigma$, let $0<\delta<C_{1}^{-1} \epsilon<C_{1}^{-1} \sigma$. Suppose $\beta$ and $\widetilde{\beta}$ are as in the lemma such that $d_{t}(\gamma(\tau), \beta(\tau))<\delta$ for all $\tau$. Since $\widetilde{\gamma}(0)=\widetilde{\beta}(0)=z_{0}$, we have $d_{e}(\widetilde{\gamma}(\tau), \widetilde{\beta}(\tau))<\epsilon$ in $\left[0, \tau_{0}\right]$ for some $\tau_{0}>0$. Let $A$ be the set $\tau$ in $[0,1]$ such that $d_{e}\left(\widetilde{\gamma}\left(\tau^{\prime}\right), \widetilde{\beta}\left(\tau^{\prime}\right)\right)<\epsilon$ for all $\tau^{\prime} \in[0, \tau]$. Then $A$ is nonempty and is open. Suppose $\tau_{k} \in A$ and $\tau_{k} \rightarrow \tau$. Then

$$
d_{e}(\widetilde{\gamma}(\tau), \widetilde{\beta}(\tau)) \leq \epsilon
$$

Since $\epsilon<\sigma, \delta<C_{1}^{-1} \sigma$, by $(3.5)$ and the fact that $\Phi_{t}(\widetilde{\gamma}(\tau))=\gamma(\tau), \Phi_{t}(\widetilde{\beta}(\tau))$ $=\beta(\tau)$, we have

$$
d_{e}(\widetilde{\gamma}(\tau), \widetilde{\beta}(\tau)) \leq C_{1} d_{t}(\gamma(\tau), \beta(\tau)) \leq C_{1} \delta<\epsilon .
$$

Hence $\tau \in A$ and $A=[0,1]$. This completes the proof of the lemma.

Apply Lemma 3.2 to $\rho=r$ and choose $\rho_{1}$ as in the lemma. Note that $\rho_{1}$ is independent of $i$ and $T$. For any $z \in D(r)$, let $\gamma^{*}(\tau), 0 \leq \tau \leq 1$, be the line segment from 0 to $z$, and let $\gamma=\Phi_{i} \circ \gamma^{*}$. By (3.2) and property (iv) for $\Phi_{t}$, there is a constant $C_{1}>0$ independent of $i$ and $T$ such that

$$
L_{i+1}(\gamma) \leq e^{-\frac{a}{2} T} L_{i}(\gamma) \leq C_{1} e^{-\frac{a}{2} T} r
$$

Now we choose $T>0$ large enough so that $C_{1} e^{-\frac{a}{2} T} r<\rho_{1}$. Then by Lemma 3.2 , there is a unique lift $\widetilde{\gamma}$ of $\gamma$ by $\Phi_{i+1}$ so that $\widetilde{\gamma}(0)=0$ and $\widetilde{\gamma} \subset D\left(\frac{r}{2}\right)$. We then define $F_{i+1}(z)=\widetilde{\gamma}(1) . F_{i+1}$ is then well-defined by the uniqueness of the lifting. We have:

Lemma 3.4. The maps $F_{i+1}$ satisfy the following:

(a) $F_{i+1}: D(r) \rightarrow D\left(\frac{r}{2}\right), F_{i+1}(0)=0$ and $\Phi_{i}=\Phi_{i+1} \circ F_{i+1}$.

(b) For each $i, F_{i+1}$ is a local biholomorphism. 
(c)

$$
b_{1}|v| \leq\left|F_{i+1}^{\prime}(0) v\right| \leq b_{2}|v|
$$

for some $0<b_{1} \leq b_{2}<1$ for all $i$ and for all vector $v \in \mathbb{C}^{n}$, where $F^{\prime}$ is the Jacobian of $F$.

(d) There exist $r>r_{1}>0$ and $0<\theta<1$ independent of $i$ such that

$$
\left|F_{i+1}(z)\right| \leq \theta|z|
$$

for all $i$ and for all $z \in D\left(r_{1}\right)$ and $F_{i+1}$ is injective on $D\left(r_{1}\right)$.

Proof. (a) follows immediately from the definition of $F_{i+1}$.

To prove (b), let us first prove that $F_{i+1}$ is continuous. Let $z_{0} \in D(r)$, $\gamma^{*}(\tau), 0 \leq \tau \leq 1$, be the line segment from 0 to $z_{0}, \gamma=\Phi_{i} \circ \gamma^{*}$ and $\widetilde{\gamma}$ is the lift of $\gamma$ by $\Phi_{i+1}$ with $\widetilde{\gamma}(0)=0$. Let $w=\widetilde{\gamma}(1)=F_{i+1}\left(z_{0}\right)$. Given $\epsilon>0$, let $\delta>0$ be as in Lemma 3.3 for $\Phi_{i+1}$.

We may assume that $\left|z_{0}\right| \leq 1-\eta$ for some $\eta>0$. Since $\Phi_{i}$ is uniformly continuous on $D(1-\eta / 2)$, there exists $\sigma>0$ such that if $\left|z_{1}-z_{2}\right|<\sigma$, $z_{1}, z_{2} \in D(1-\eta / 2)$, then $d_{i}\left(\Phi_{i}\left(z_{1}\right), \Phi_{i}\left(z_{2}\right)\right)<\delta$.

Moreover, it is easy to see that we can find $\delta^{\prime}>0$ such that if $\left|z_{0}-\zeta\right|<$ $\delta^{\prime}$, then the ray $\beta^{*}$ defined on $[0,1]$ from 0 to $\zeta$ satisfies $\left|\gamma^{*}(\tau)-\beta^{*}(\tau)\right|<\sigma$ and $\beta^{*} \subset D(1-\eta / 2)$. Let $\zeta$ be such a point in $D(r)$ with $\beta^{*}$ as above and let $\beta=\Phi_{i} \circ \beta^{*}$. Hence we have

$$
d_{i+1}(\gamma(\tau), \beta(\tau)) \leq d_{i}(\gamma(\tau), \beta(\tau))<\delta .
$$

By Lemma 3.3, if $\widetilde{\beta}$ is the lift of $\beta$ by $\Phi_{i+1}$ with $\widetilde{\beta}(0)=0$, then $\mid \widetilde{\gamma}(1)-$ $\widetilde{\beta}(1) \mid<\epsilon$. That is to say, $\left|F_{i+1}\left(z_{0}\right)-F_{i+1}(\zeta)\right|<\epsilon$ and $F_{i+1}$ is continuous.

Now it is easy to see that $F_{i+1}$ is a local biholomorphism. In fact, suppose $F_{i+1}(z)=w$ and suppose $\Phi_{i}(z)=x$ and $\Phi_{i+1}(w)=y$. Let $\epsilon_{1}>0$ be such that $\Phi_{i}$ and $\Phi_{i+1}$ are biholomorphisms when restricted on $D\left(z, \epsilon_{1}\right)$ and $D\left(w, \epsilon_{1}\right)$, respectively. Since $F_{i+1}$ is continuous, we can find $0<\delta_{1}<\epsilon_{1}$ such that $F_{i+1}\left(D\left(z, \delta_{1}\right)\right) \subset D\left(w, \epsilon_{1}\right)$. Since $\Phi_{i}=\Phi_{i+1} \circ F_{i+1}$, we have

$$
F_{i+1}=\Phi_{i+1}^{-1} \circ \Phi_{i}
$$

on $B_{e}\left(z, \delta_{1}\right)$. Hence $F_{i+1}$ is a local biholomorphism.

To prove (c), by properties (ii), (iii) of $\Phi_{t}$, the fact that $F_{i+1}=\Phi_{i+1}^{-1} \circ \Phi_{i}$ near the origin, the fact that $R_{i \bar{\jmath}}(t)$ is uniformly bounded and (3.1), it is easy to see that (c) is true. 
To prove (d), by gradient estimates, we have

$$
F_{i+1}(z)=F_{i+1}(0)+F_{i+1}^{\prime}(0) z+H_{i}(z)=F_{i+1}^{\prime}(0) z+H_{i}(z)
$$

where $\left|H_{i}^{\prime}(z)\right| \leq C|z|$ for some constant $C$ independent of $i$ on $D\left(\frac{1}{2} r\right)$, say. Hence by (c), there exist $r>r_{1}>0$ and $1>\eta>0$, independent of $i$ such that $F_{i+1}: D\left(r_{1}\right) \rightarrow D\left(r_{1}\right)$ so that

$$
\left|F_{i+1}(z)\right| \leq \theta|z|
$$

for all $i$ and for all $z \in D\left(r_{1}\right)$ and $F_{i+1}$ is injective on $D\left(r_{1}\right)$.

By this lemma and Theorem 2.6, using the method in $[9, \S 5]$, see also $[19,30]$, we can prove the following:

Lemma 3.5. Let $F_{i}$ be as in Lemma 3.4. There exist biholomorphisms $G_{i}$ on $\mathbb{C}^{n}$ and polynomial maps $T_{i}$ with the following properties:

(a) $T_{i}(0)=0, T_{i}^{\prime}(0)=I d$, and $\sup _{z \in D(1)}\left|T_{i}(z)\right| \leq C_{1}$ for some constant $C_{1}$ independent of $i$.

(b) $G_{i}(0)=0$ and for all open sets, $U$ containing the origin

$$
\bigcup_{k=1}^{\infty}\left(G_{k} \circ \cdots \circ G_{1}\right)^{-1}(U)=\mathbb{C}^{n} .
$$

(c) There exist $0<r_{2}<r_{1}$ and $C_{2}>0$ independent of $k \geq 1$ such that

$$
G_{k+1}^{-1} \circ G_{k+2}^{-1} \circ \cdots \circ G_{k+l}^{-1} \circ T_{k+l} \circ F_{k+l} \circ \cdots \circ F_{k+2} \circ F_{k+1}
$$

converges uniformly on $D\left(r_{2}\right)$ as $l \rightarrow \infty$ to an injective holomorphic map $\Psi_{k}$ such that

$$
D\left(C_{2}^{-1} r_{2}\right) \subset \Psi_{k}\left(D\left(r_{2}\right)\right) \subset D\left(C_{2} r_{2}\right) .
$$

(d) By choosing $r_{2}$ smaller if necessary, we may have that $T_{i}$ is injective on $D\left(r_{2}\right)$ such that $T_{i}^{-1}$ is defined on $D\left(r_{2}\right)$ and $T_{i}^{-1}\left(D\left(r_{2}\right)\right) \subset D(r)$ for all $i$.

\section{Proof of the main theorem}

We are now ready to prove Theorem 1.5. By the remark at the beginning of $\S 2$, we may assume that $M$ is simply connected and $g(t)$ has positive Ricci curvature for all $t$. 
Let $\Phi_{i}$ be as in the previous section so that one can define $F_{i}$ as in Lemma 3.4. Let $G_{i}, T_{i}, r_{2}, C_{1}$ and $C_{2}$ be as in Lemma 3.5. We want to construct a biholomorphism from $\mathbb{C}^{n}$ onto $M$ as follows: Let $\Omega_{i}=\left(G_{i} \circ \cdots \circ\right.$ $\left.G_{1}\right)^{-1}\left(D\left(r_{2}\right)\right)$ and define

$$
S_{i}=\Phi_{i} \circ T_{i}^{-1} \circ G_{i} \circ \cdots \circ G_{1}
$$

which is defined on $\Omega_{i}$ by Lemma 3.5.

Theorem 1.5 will be proved if we can prove that $S_{i}$ converges to a bihololomorphism from $\mathbb{C}^{n}$ onto $M$. We will prove this in several steps as described in the following lemmas.

Lemma 4.1. For all $z \in \mathbb{C}^{n}, \lim _{i \rightarrow \infty} S_{i}(z)=S(z)$ exists.

Proof. Let $k$ be fixed and consider $U_{k}=\left(G_{k} \circ \cdots \circ G_{1}\right)^{-1}\left(\frac{1}{2 C_{2}} D\left(r_{2}\right)\right)$, where $C_{2}, r_{2}$ are as in Lemma 3.5(c). Let $\Psi_{k}$ as in Lemma 3.5(c). Since the convergence in the lemma is uniform in $D\left(r_{2}\right)$, and $G_{k+1}^{-1} \circ G_{k+2}^{-1} \circ \cdots \circ G_{k+l}^{-1} \circ$ $T_{k+l} \circ F_{k+l} \circ \cdots \circ F_{k+2} \circ F_{k+1}$ and $\Psi_{k}$ are injective, we can find $0<\rho<r_{2}$ and $l_{0}$ such that

$$
\begin{aligned}
& G_{k+1}^{-1} \circ G_{k+2}^{-1} \circ \cdots \circ G_{k+l}^{-1} \circ T_{k+l} \circ F_{k+l} \circ \cdots \circ F_{k+2} \circ F_{k+1}(D(\rho)) \\
& \quad \supset D\left(\frac{1}{2 C_{2}} r_{2}\right)
\end{aligned}
$$

if $l \geq l_{0}$. Hence for every $l \geq l_{0}$ we have: for every $z \in U_{k}$, there exists $\zeta_{l} \in D(\rho)$ such that$$
G_{1}^{-1} \circ \cdots \circ G_{k}^{-1} \circ G_{k+1}^{-1} \circ \cdots \circ G_{k+l}^{-1} \circ T_{k+l} \circ F_{k+l} \circ \cdots \circ F_{k+2} \circ F_{k+1}\left(\zeta_{l}\right)=z \text {. }
$$

Hence

$$
\begin{aligned}
S_{k+l}(z) & =\Phi_{k+l} \circ T_{k+l}^{-1} \circ G_{k+l} \circ \cdots \circ G_{1}(z) \\
& =\Phi_{k+l} \circ F_{k+l} \circ \cdots \circ F_{k+2} \circ F_{k+1}\left(\zeta_{l}\right) \\
& =\Phi_{k}\left(\zeta_{l}\right) .
\end{aligned}
$$

Take two such subsequences $\zeta_{l_{j}}$ and $\zeta_{l_{j}^{\prime}}$ such that $\zeta_{l_{j}} \rightarrow w$ and $\zeta_{l_{j}^{\prime}}=w^{\prime}$ as $j \rightarrow \infty$ with $w, w^{\prime} \in \overline{D(\rho)}$. Since the convergence in Lemma 3.5 is uniform, by (4.2) we have

$$
G_{1}^{-1} \circ \cdots \circ G_{k}^{-1} \circ \Psi_{k}(w)=z=G_{1}^{-1} \circ \cdots \circ G_{k}^{-1} \circ \Psi_{k}\left(w^{\prime}\right) .
$$


Hence we must have $w=w^{\prime}$ and so $\zeta_{l} \rightarrow w$ as $l \rightarrow \infty$. By (4.3), we have

$$
\begin{aligned}
\lim _{l \rightarrow \infty} S_{k+l}(z) & =\Phi_{k}(w) \\
& =\Phi_{k} \circ \Psi_{k}^{-1} \circ G_{k} \circ \cdots \circ G_{1}(z) .
\end{aligned}
$$

Hence $S=\lim _{i \rightarrow \infty} S_{i}$ exists on $U_{k}$. By Lemma 3.5, $\bigcup_{k} U_{k}=\mathbb{C}^{n}$, from this the lemma follows.

Lemma 4.2. $S$ is a local biholomorphic map from $\mathbb{C}^{n}$ into $M$.

Proof. This follows immediately from (4.4).

Lemma 4.3. For any $\epsilon>0, \bigcup_{k} \Phi_{k}(D(\epsilon))=M$.

Proof. Since the Ricci curvature of $g(0)$ is positive, for any $R>0$, we have $R_{i \bar{\jmath}}(x, 0) \geq a g_{i \bar{\jmath}}(x, 0)$ for some $a>0$ for all $x \in B_{0}(p, R)$, which is the geodesic ball with respect to $g(0)$. Let $\lambda_{i}(x, t)$ be the eigenvalues of $R_{i \bar{\jmath}}(x, t)$ with respect to $g(t)$. Then $\lambda_{i}(x, t) \leq C$ for some constant $C$ independent of $x$ and $t$. On the other hand, by Proposition 2.6, we have $\prod_{i=1}^{n} \lambda_{i}(x, t) \geq a^{n}$ for $x \in B_{0}(p, R)$. Hence there exists $b>0$ independent of $t$ such that

$$
R_{i \bar{\jmath}}(x, t) \geq b g_{i \bar{\jmath}}(x, t)
$$

for all $t \geq 0$ and $x \in B_{0}(p, R)$. By the Kähler-Ricci flow equation, we conclude that

$$
B_{0}(p, R) \subset B_{t}\left(p, e^{-\frac{b+\kappa}{2} t} R\right) .
$$

From this and Lemma 3.1 the lemma follows.

Lemma 4.4. $S$ is surjective.

Proof. From the proof of Lemma 4.1, we conclude that

$$
S\left(\mathbb{C}^{n}\right) \supset \Phi_{k} \circ \Psi_{k}^{-1}\left(D\left(\frac{1}{2 C_{2}} r_{2}\right)\right)
$$

for all $k$. From this, Lemma 4.3 and the proof of Lemma 3.5 (c) (see $[9, \S 5]$ ), it is easy to see that $S\left(\mathbb{C}^{n}\right)=M$.

Lemma 4.5. $S$ is injective. 
Proof. Suppose there exists distinct $z_{1}, z_{2} \in \mathbb{C}^{n}$ such that $S\left(z_{1}\right)=S\left(z_{2}\right)=q$. Let $\sigma(\tau), 0 \leq \tau \leq 1$ be the line segment from $z_{1}$ to $z_{2}$. Let $\gamma=S \circ \sigma$. Then $\gamma$ is a smooth closed curve in $M$ starting from $q$. Since $M$ is simply connected, we can find a smooth homotopy $\alpha(s, \tau), 0 \leq s, \tau \leq 1$, with $\alpha(0, \tau)=\gamma(\tau)$, $\alpha(1, \tau)=q$ (the constant map), and $\alpha(s, 0)=\alpha(s, 1)=q$ for all $s$.

By Lemma 3.5, there exists $0<\rho<r_{2}$ and $\eta>0$ which are independent of $i$ such that $\Psi_{i}^{-1}$ is defined on $D(\eta)$ and

$$
\Psi_{i}^{-1}(D(\eta)) \subset D\left(\frac{1}{8} \rho\right)
$$

For $\rho>0$ let $\rho_{1}>0$ be such that Lemma 3.2 is true. By the proof of Lemma 4.3 , there exists $k_{0}$ such that if $k \geq k_{0}$, then $q \in B_{k}\left(p, \rho_{1}\right)$ and $L_{k}(\alpha(s, \cdot))<$ $\rho_{1}$ for all $s$, where $B_{k}\left(p, \rho_{1}\right)$ is the geodesic ball of radius $\rho_{1}$ at $p$ relative to $g(k T)$, and $L_{k}$ is the length with respect to $g(k T)$. We may also assume that $\sigma \subset G_{1}^{-1} \circ \cdots \circ G_{k}^{-1}(D(\eta))$, provided $k_{0}$ is large enough.

Now fix $k \geq k_{0}$. Let $\widetilde{\gamma}=\Psi_{k}^{-1} \circ G_{k} \circ \cdots \circ G_{1} \circ \sigma$. Then $\widetilde{\gamma} \subset D\left(\frac{\rho}{8}\right)$ by (4.5) and it is a lift of $\gamma$ by $\Phi_{k}$ by (4.4). Moreover, if $\widetilde{\gamma}(0)=w_{1}$, and $\widetilde{\gamma}(1)=w_{2}$, then $w_{1} \neq w_{2}$ because $G_{i}$ and $\Psi_{k}$ are injective. Since $\Phi_{k}\left(w_{1}\right)=$ $\Phi_{k}\left(w_{2}\right)=q \in B_{k}\left(p, \rho_{1}\right)$, by Lemma 3.2, for any $s$, there is a lift $\widetilde{\beta}_{s}(\tau)$ of $\alpha(s, \tau)$ by $\Phi_{k}$ such that $\widetilde{\beta}_{s}(0)=w_{1}$ and $\widetilde{\beta}_{s} \subset D\left(\frac{\rho}{2}\right)$.

We claim that $\widetilde{\beta}_{s}(1)=w_{2}$. Let $\epsilon>0$ be such that $\Phi_{k}$ is a biholomorphism onto its image when restricted on $B_{e}\left(w_{2}, \epsilon\right)$. For such $\epsilon>0$, let $\delta>0$ be as in Lemma 3.3. On the other hand, let $\xi>0$ be such that

$$
d_{k}\left(\alpha\left(s_{1}, \tau\right), \alpha\left(s_{2}, \tau\right)\right)<\delta
$$

for all $\tau$, if $\left|s_{1}-s_{2}\right| \leq \xi$.

Hence by Lemma 3.3, if $0 \leq s \leq \xi$ then $\widetilde{\beta}_{s}(1) \in B_{e}\left(w_{2}, \epsilon\right)$. But $\Phi_{k}\left(\widetilde{\beta}_{s}(1)\right)$ $=\alpha(s, 1)=q$, and $\Phi_{k}$ is injective on $B_{e}\left(w_{2}, \epsilon\right)$. Thus we have $\widetilde{\beta}_{s}(1)=w_{2}$ for $0 \leq s \leq \xi$. In particular, $\widetilde{\beta}_{\xi}(1)=w_{2}$. By (4.6) and Lemma 3.3, we can argue as before and conclude that $\widetilde{\beta}_{2 \xi}(1)=w_{2}$. Continue in this way, we have that $\widetilde{\beta}_{1}(1)=w_{2}$. On the other hand, $\widetilde{\beta}_{1}$ is a lift of $\alpha(1, \cdot)$. Hence $\Phi_{k}\left(\widetilde{\beta}_{1}(\tau)\right)=q$ for all $\tau$. Since $w_{1} \neq w_{2}$, there is $\tau$ with $\tilde{\beta}_{1}(\tau) \neq w_{2}$ and $\tilde{\beta}_{1}(\tau)-w_{2} \mid \leq \frac{\epsilon}{2}$. This is impossible because $\Phi_{k}$ is a injective on $B_{e}\left(w_{2}, \epsilon\right)$.

Theorem 1.5 now follows from Lemmas 4.2, 4.4 and 4.5.

Corollary 4.6. Let $(M, g(t))$ be as in Theorem 1.5 and assume that $g(0)$ has positive Ricci curvature at some point $p \in M$. Then $M$ is biholomorphic to $\mathbb{C}^{n}$. In particular, if $(M, g)$ is as in Theorem 1.2 and the Ricci curvature of $g$ is positive at some point, then $M$ is biholomorphic to $\mathbb{C}^{n}$. 
Proof. Let $(M, g(t))$ as in Theorem 1.5. We begin by proving that $M$ has finite fundamental group. Suppose $M$ has infinite fundamental group and let $\widetilde{M}$ be the universal cover of $M$. Then $\widetilde{M}$ contains infinitely many preimages $p_{0}, p_{1}, \ldots$ of $p \in M$, and for simplicity we will denote $p_{0}$ simply by $p$. We may also pull $g(t)$ back to obtain a solution to (1.6) on $\widetilde{M}$, and we denote this solution again by $g(t)$. Note that by $[6],(\widetilde{M}, g(t))$ must have positive Ricci curvature on all of $\widetilde{M}$ and for all $-\infty<t<\infty$ since $\widetilde{M}$ is simply connected and $\operatorname{Ric}(p, 0)>0$ by assumption. Now for $(\widetilde{M}, g(t), p)$ consider $\Phi_{t}: D(r) \rightarrow \widetilde{M}$ be as in Proposition 2.1. Then by choosing $r$ smaller, if necessary, we may have the following for all $t \geq 0$ :

(a1) $R i c_{t} \geq c>0$ in $D(r)$ with respect to $\Phi_{t}^{*}(g(t))$ for some constant $c$.

(a2) There exists $0<\delta<1$ such that any point in $D(\delta r)$ can be joined by a geodesic from $p$ in $D\left(\frac{2}{3} r\right)$ with respect to $\Phi_{t}^{*}(g(t))$.

(a3) There exists $C>1$ such that $B_{t}\left(p, \frac{1}{C} \rho\right) \subset \Phi_{t}(D(\rho)) \subset B_{t}(p, C \rho)$ for all $\rho<r$.

(a1) follows from Propositions 2.1 and 2.3 and the fact that the covariant derivatives of the curvature tensor is bounded. (a2) follows from Proposition 2.1 and the construction of $\Phi_{t}$ through the exponential map so that the injectivity radius of $\Phi_{t}^{*}(g(t))$ at the origin is uniformly bounded below by a positive constant. (a3) follows from the proof of Lemma 3.1.

Note that we must have $\lim _{k \rightarrow \infty} d_{g(0)}\left(p_{k}, p\right)=\infty$. Also, by the proof of Lemma 4.3 , we know that the sets $B_{t}(p, \epsilon r)$, where $\epsilon=\frac{\delta}{C}$, exhaust $M$ as $t \rightarrow \infty$. Thus, we can find a subsequence of $p_{k}$, also denoted by $p_{k}$, and a sequence $t_{k} \rightarrow \infty$ such that $p_{k} \in \partial B_{t_{k}}(p, \epsilon r)$.

Now consider the sequence of pull-backs $\Phi_{t_{k}}^{*}\left(g\left(t_{k}+t\right)\right.$ on $D(r)$. Then by Theorem 2.4 we may assume the sequence converges on $D(r)$ to some $h(t)$ satisfying $R_{i \bar{\jmath}}+\kappa h_{i \bar{\jmath}}=f_{i \bar{\jmath}}$ at $t=0$, where $f$ is smooth, $f_{i j}=0$ and $\nabla f(0)=0$. Now for each $k$, let $z_{k}$ be an inverse image of $p_{k}$ under $\Phi_{t_{k}}$ (which exists by our construction of $p_{k}$ ). Then $C^{-2} \delta \leq\left|z_{k}\right| \leq \delta r$ for all $k$ by (a3), and hence we may also assume that $z_{k} \rightarrow w$, where $C^{-2} \delta r \leq|w| \leq \delta r$.

Moreover, if $\sigma$ is the covering map that maps $p_{k}$ to $p$, then $g(t)=$ $\sigma^{*}(g(t))$.

For any $p_{k}$, let $F: \widetilde{M} \rightarrow \widetilde{M}$ be a deck transformation taking $p_{k}$ to $p$. Then for every $t, F$ is an isometry with respect to $g\left(t_{k}+t\right)$. Hence, for every $k, \Phi_{t_{k}}^{*}\left(g\left(t_{k}+t\right)\right)$ is the 'same' at the origin $o$ and $z_{k}$, and in particular $R(0, t)=R\left(z_{k}, t\right)$ for all $t$. Thus, by letting $k \rightarrow \infty$, we have that $R(w, t)$ is constant in time since $R(o, t)$ is constant in time. Furthermore, by the proof of Theorem 2.4, we may have that $\nabla f(w)=0$. 
Now by (a2), we can join $o$ to $w$ by a geodesic w.r.t. $h(0)$ parametrized by arc-length. As in [18: Proof of Theorem 20.1], we may compute in real coordinates $x_{\alpha}$ as follows:

$$
\frac{d^{2} f}{d s^{2}}=D_{\alpha} D_{\beta} f \cdot \frac{d x_{\alpha}}{d s} d x_{\beta}>0
$$

since $R_{i \bar{\jmath}}>0, R_{i \bar{\jmath}}+\kappa h_{i \bar{\jmath}}=f_{i \bar{\jmath}}$ and $f_{i j}=0$. But this is impossible as

$$
\frac{d f}{d s}=0
$$

at the end points $o$ and $w$. We have thus proved, by contradiction, that the fundamental group of $M$ must be finite.

It now follows by a well-known result (see [2, p. 35 and p. 40]) that $M$ must be simply connected, and thus biholomorphic to $\mathbb{C}^{n}$ by the conclusion of Theorem 1.5. This completes the proof of the corollary.

\section{Acknowledgments}

The authors would like to thank Huai-Dong Cao, David Glickenstein, Richard Hamilton, Hung-Hsi Wu and Shing-Tung Yau for helpful discussions. They would also like to thank Lei Ni for informing them that the second part of Corollary 4.6 can also be proved using his results in ([24], Corollary 5.2).

\section{References}

[1] L. Barreira and Y. B. Pesin, Lyapunov exponents and smooth ergodic theory, University Lecture Series v. 23, American Mathematical Society, 2001, Brown.

[2] K. S. Brown, Cohomology of groups, Springer-Verlag, 1982.

[3] R. Bryant, Gradient Kähler Ricci solitons, arXiv eprint 2004. arXiv:math.DG/0407453.

[4] H.-D. Cao, On Harnack's inequality for the Kähler-Ricci flow, Invent. Math. 109 (1992), 247-263.

[5] H.-D. Cao, Limits of solutions to the Kähler-Ricci flow, J. Differ. Geom. 45 (1997), 257-272. 
[6] H.-D. Cao, On dimension reduction in the Kähler-Ricci flow, Comm. Anal. Geom. 12 (2004), 305-320.

[7] A. Chau and L.-F. Tam, Gradient Kähler-Ricci soliton and a uniformization conjecture, arXiv eprint 2002. arXiv:math.DG/0310198.

[8] A. Chau and L.-F. Tam, A note on the uniformization of gradient Kähler-Ricci solitons, Math. Res. Lett. 12 (2005), no. 1, 19-21.

[9] A. Chau and L.-F. Tam, On the complex structure of Kähler manifolds with non-negative curvature, J. Differ. Geom. 73 (2006), 491-530.

[10] B. L. Chen and X. P. Zhu, On complete noncompact Kähler manifolds with positive bisectional curvature, Math. Ann. 327 (2003), 1-23.

[11] B. L. Chen, S. H. Tang and X. P. Zhu, A uniformization theorem of complete noncompact Kähler surfaces with positive bisectional curvature, J. Differ. Geom. 67 (2004), 519-570.

[12] B. L. Chen and X. P. Zhu, Positively curved complete noncompact Kähler Manifolds, arXiv eprint 2002. arXiv:math.DG/0211373.

[13] B. L. Chen and X. P. Zhu, Volume growth and curvature decay of positively curved Kähler manifolds, Q. J. Pure Appl. Math. 1 (2005), no. 1, 68-108.

[14] B. Chow, D. Glickenstein and P. Lu, Metric transformations under collapsing of Riemannian manifolds, Math. Res. Lett. 10 (2003), 737-746.

[15] B. Chow, D. Glickenstein and P. Lu, Collapsing sequences of solutions to the Ricci flow on 3-manifolds with almost nonnegative curvature, Math. Z. 254 (2006), no. 1, 1-28.

[16] D. Glickenstein, Precompactness of solutions to the Ricci flow in the absence of injectivity radius estimates, Geom. Topol. 7 (2003), 487-510.

[17] R. S. Hamilton, A compactness property for solutions of the Ricci flow, Amer. J. Math. 117 (1995), 545-572.

[18] R. S. Hamilton, Formation of singularities in the Ricci Flow, Surv. Differ. geom. II (1995), 7-136.

[19] M. Jonsson and D. Varolin, Stable manifolds of holomorphic diffeomorphisms, Invent. Math. 149 (2002), 409-430.

[20] J. Lott, On the long-time behavior of type-III Ricci flow solutions, arXiv eprint 2005. arxiv:math.DG/0509639.

[21] N. Mok, An embedding theorem of complete Kähler manifolds of positive bisectional curvature onto affine algebraic varieties, Bull. Soc. Math. France. 112 (1984), 179-258. 
[22] N. Mok, Y.-T. Siu and S.-T. Yau, The Poincaré-Lelong equation on complete Kähler manifolds, Comp. Math. 44 (1981), 183-218.

[23] L. Ni, Vanishing theorems on complete Kähler manifolds and their applications, J. Differ. Geom. 50 (1998), 89-122.

[24] L. Ni, A monotonicity formula on complete Kähler manifolds with nonnegative bisectional curvature, J. Amer. Math. Soc. 17 (2004), 909-946.

[25] L. Ni, Ancient solutions to Kähler-Ricci flow, Math. Res. Lett. 12 (2005), 633-653.

[26] L. Ni, Y.-G. Shi and L.-F. Tam, Poisson equation, Poincaré-Lelong equation and curvature decay on complete Kähler manifolds, J. Differ. Geom. 57 (2001), 339-388.

[27] L. Ni and L.-F. Tam, Kähler-Ricci flow and the Poincaré-Lelong equation, Comm. Anal. Geom. 12 (2004), 111-141.

[28] L. Ni and L.-F. Tam, Plurisubharmonic functions and the structure of complete Kähler manifolds with nonnegative curvature, J. Differ. Geom. 64 (2003), 457-524.

[29] M. H. Protter and H. F. Weinberger, Maximum principles in differential equations, Prentice Hall, 1967.

[30] J. P. Rosay and W. Rudin, Holomorphic maps from $\mathbb{C}^{n}$ to $\mathbb{C}^{n}$, Trans. AMS 310 (1988), 47-86.

[31] W.-X. Shi, Ricci deformation of the metric on complete noncompact Riemannian manifolds, J. Differ. Geom. 30 (1989), 223-301.

[32] W.-X. Shi, Ricci deformation of the metric on complete noncompact Kähler manifolds, PhD thesis, Harvard University, 1990.

[33] W.-X. Shi, Complete noncompact Kähler manifolds with positive holomorphic bisectional curvature, Bull. Amer. Math. Soc. (N. S.) 23 (1990), 337-400.

[34] W.-X. Shi, Ricci flow and the uniformization on complete non compact Kähler manifolds, J. Differ. Geom. 45 (1997), 94-220.

[35] G. Tian and S.-T. Yau, Complete Kähler manifolds with zero Ricci curvature. I., J. Amer. Math. Soc. 3 (1990), 579-609.

[36] S.-T. Yau, A review of complex differential geometry, Proc. Sympos. Pure Math., 52 Part 2 (1991), 619-625.

[37] R. Ye, Curvature estimates for the Ricci flow II, arXiv:math. DG/0509143. 


\author{
WATERLOO UNIVERSITY \\ Department of Pure Mathematics \\ 200 UNIVERSITY AVENUE, WATERLOO \\ ON N2L 3G1, CANADA \\ E-mail address: a3chau@math.uwaterloo.ca \\ Department of MATHEMATics \\ The Chinese University of Hong Kong \\ Shatin, Hong Kong, China \\ E-mail address: lftam@math.cuhk.edu.hk
}

ReCEIVED May 3, 2006 